\title{
Fabrication and Healing of Faceted Nanopores in Magnesium
}

\author{
Shujing $\mathrm{Wu}^{1}$, Huaping Sheng ${ }^{1}$, Chun $\mathrm{Liu}^{1}$, Fan Cao ${ }^{1}$, Yu Liu ${ }^{1}$, He Zheng ${ }^{1}$, Dongshan Zhao and Jianbo \\ Wang $^{1}$
}

1. School of Physics and Technology, Center for Electron Microscopy and MOE Key Laboratory of Artificial Micro- and Nano-structures, Wuhan University, Wuhan 430072, China

Solid-state nanopores have been extensively investigated due to many biological engineering and scientific applications, such as for rapid electrical detection and analysis of biopolymers, powerful DNA detection and genome sequencing technology [1-2]. Herein, we demonstrate that faceted nanopores with various shapes can be successfully fabricated in magnesium via focused electron beam (e-beam) technology inside transmission electron microscope (TEM). Associated with orientation, the nanopore shapes varied when manipulating the e-beam irradiation direction (Figure 1). The characteristics of nanopore shapes can be explained by Wulff construction, and the crystallographic planes corresponding to the edges of nanopores are also discussed in detail [3].

Furthermore, employing the in situ high-resolution transmission electron microscopy (HRTEM) techniques, we obtained the first experimental evidence that the nanopores would continuously shrink and finally disappear when the e-beam was spread out (Figure 2), as evidenced by the layer-by-layer growth of atomic planes at the nanopore periphery [4]. When the e-beam was turned off, the nanopore would retain its shape, suggesting an e-beam-assisted healing mechanism. Hence, by manipulating the e-beam (e.g., irradiation direction and duration), the nanopore shape and size could be effectively controlled along different directions. Our results provide an important insight into the nanopore patterning in metallic materials and are of fundamental importance concerning the relevant applications, such as nanopore-based sensor, etc. The healing properties of $\mathrm{Mg}$ have extended its possible applications in irradiated environments, such as in outer space.

The TEM specimens were first prepared by cutting into $1 \mathrm{~mm}$ thick slices from original bulk material, and then thinning through polishing with abrasive paper to a thickness of approximately $50 \mu \mathrm{m}$. In subsequence, the samples were punched into disks of $3 \mathrm{~mm}$ in diameter, which were then electro-chemically polished. The specimens were ion-milled employing a Gatan precision ion polishing system. Afterwards, selected area electron diffraction (SAED) patterns were obtained utilizing the JEM-2010 (HT) (HT: high angle tilt). In situ HRTEM observation was performed inside the JEM-2010 FEF (UHR) (FEF: field emission gun and in-column X-type energy filter; UHR pole-piece: ultrahigh resolution with a Scherzer resolution of $0.19 \mathrm{~nm}$ ). Both microscopes were operated at an accelerating voltage of $200 \mathrm{kV}$ [5].

[1] H Chang et al, Nano Lett 4 (2004), p. 1551.

[2] IM Derrington et al, Proc Natl Acad Sci USA 107 (2010), p. 16060.

[3] S Wu et al, Appl Phys Lett 103 (2013), p. 243101.

[4] H Zheng et al, Sci Rep 3 (2013), p. 1920.

[5] This work was supported by the 973 Program (2011CB933300), the National Natural Science

Foundation of China $(51071110,51271134,40972044$, J1210061, 51371130), the China MOE NCET Program (NCET-07-0640), MOE Doctoral Fund (20090141110059), the Fundamental Research Funds for the Central Universities, and the China Postdoctoral Science Foundation (2013M540602). 

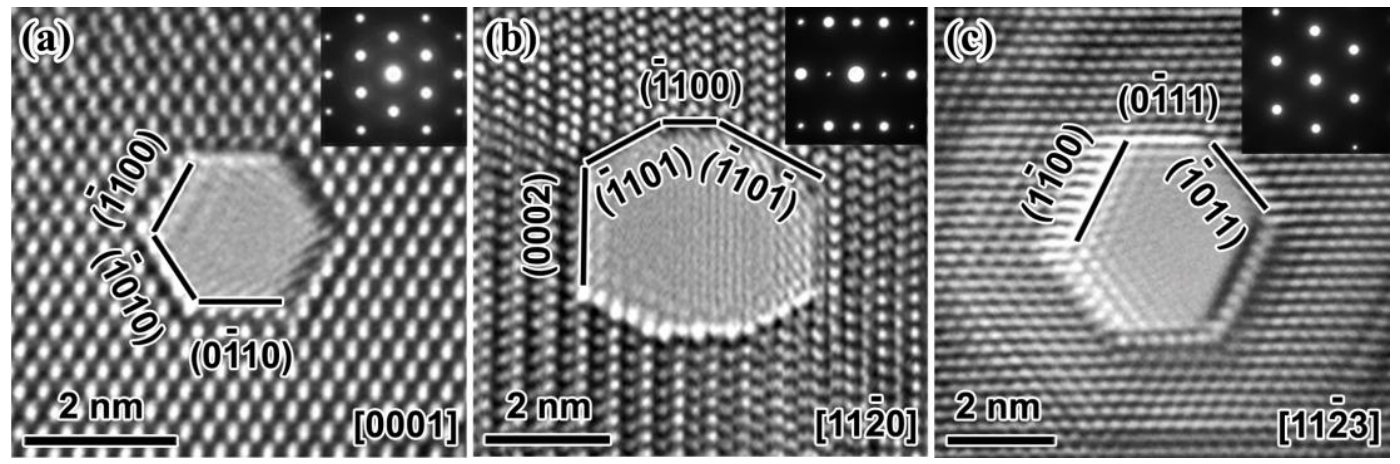

Figure 1. Fabrication of faceted nanopores in Mg. HRTEM images of nanopores along the (a) [0001], (b) [11-20], and (c) [11-23] zone axes, correspondingly. The insets in (a), (b), and (c) show the SAED patterns of the corresponding regions, respectively.
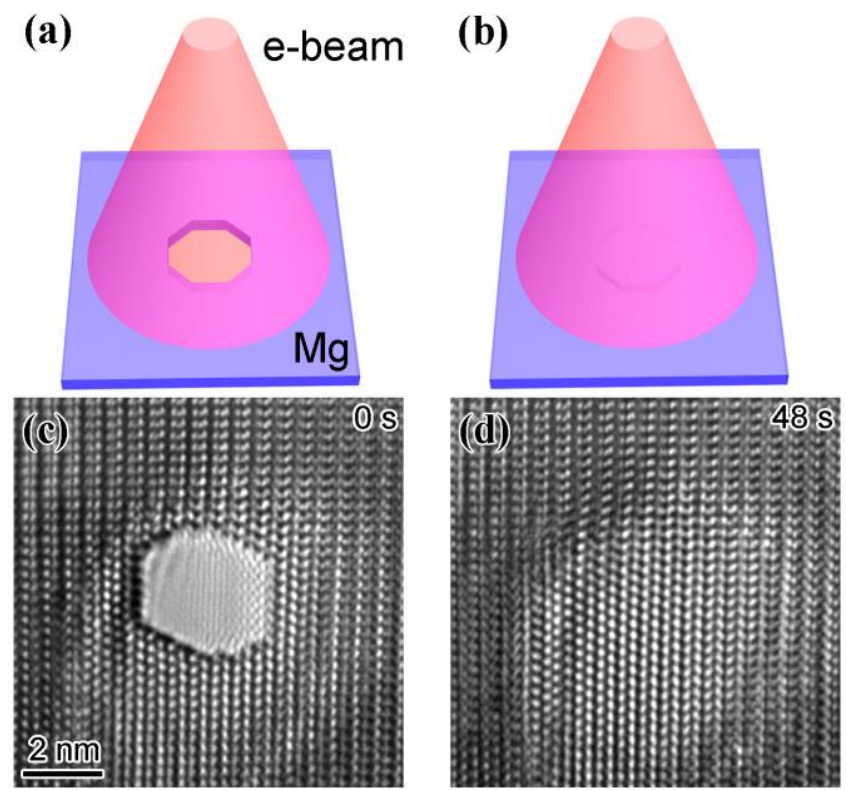

Figure 2. E-beam assisted healing of faceted nanopores in Mg along the [11-20] zone axis. (a-b) A schematic illustration showing the healing of an individual nanopore under wide-field e-beam irradiation. (c-d) Time-lapsed images illustrating the experimental observations of the healing process of a nanopore with an original size of $3.3 \mathrm{~nm}$. 